\title{
Comparación de la efectividad en la protección de cultivos de tomates con insecticidas orgánicos a base de: ajo (allium sativum) y Nim (azadirachta indica)
}

\section{Comparison of effectiveness in the protection of tomato crops with organic insecticides based on: garlic(allium sativum) and Nim(azadirachta indica)}

\author{
Abdiel Bordones ${ }^{l}$, Norvis DeGracia ${ }^{l}$, Domiciano Díaz, Reinier Rodríguez $^{l}$, Alma Chen ${ }^{2 *}$ \\ ${ }^{1}$ Licenciatura en Ingeniería Electromecánica - Centro Regional de Veraguas - Universidad Tecnológica de Panamá, \\ ${ }^{2}$ Centro Regional de Veraguas - Universidad Tecnológica de Panamá
}

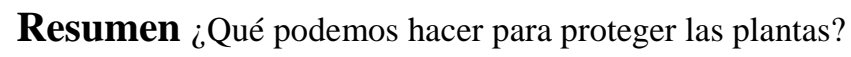

El objetivo de esta investigación consiste en la elaboración de dos insecticidas orgánicos a base de ajo y Nim, además realizar una comparación para determinar la efectividad en el control y eliminación de insectos plagas en cultivos de tomate industrial. Dichos insecticidas son una alternativa ecológica para el medio ambiente y la salud de los productores y consumidores, debido a que no afecta el ecosistema y el producto final no contiene residuos de agroquímicos. Esta opción combina y aprovecha aquellas ventajas que brindan las plantas, a través de sus componentes y propiedades activas que son efectivas para la elaboración de insecticidas. Por su alta efectividad en el control de enfermedades tropicales, insectos y el alto rendimiento por hectáreas, composición y modo de acción.

El tomate es uno de los rubros que se produce en la región, sus costos de producción son bajos, posee alta calidad nutricional en vitaminas y minerales. En sus etapas de desarrollo es afectado por numerosas plagas como minadoras, chupadoras, masticadores etc. y es atacada por enfermedades diversas debido a que los plantones están a campo abierto y su periodo de cosecha es muy largo (3 a 5 meses). El modo de acción de ambos insecticidas en estas plagas es por ingestión e inhalación.

La principal sustancia presente en el extracto de las hojas de Nim es la Azadiractina la cual tiene una acción repelente contra insectos plaga y además rompe el ciclo vital impidiendo su multiplicación [1], para el caso del ajo, su principal sustancia activa para la elaboración de insecticidas es la Alina que cuando es liberada interactúa con una enzima llamada alinasa y genera la alicina que es la sustancia que genera el olor característico del ajo y con el cual los insectos son repelados de la planta infectada. [2]

El proceso de la elaboración de estos insecticidas inicia con el procesamiento y la extracción de las sustancias de ambos productos; posteriormente se aplica en los plantones.

Luego de la aplicación de los insecticidas se observó que los plantones afectados por los insectos, mejoraron su forma y tuvieron un mejor crecimiento. El insecticida a base de Nim tuvo un $100 \%$ de efectividad en los plantones donde fue probado, en cambio la efectividad del ajo fue de $66.7 \%$ en los plantones tratados, el modo de actuar del Nim en la planta es introduciéndose a la savia y el del ajo a través de sus raíces, concluimos que el Nim es mejor producto para la elaboración de insecticidas orgánicos.

Palabras claves Nim, insecticida, agricultura ecológica, tóxico, azadiractina, plagas, insectos, alicina, alinasa.

\begin{abstract}
What can we do to protect plants?
The objective of this research is the elaboration of two organic insecticides based on garlic and neem, in addition to making a comparison to determine the effectiveness in the control and elimination of insect pests in industrial tomato crops. These insecticides are an ecological alternative for the environment and the health of producers and consumers, because it does not affect the ecosystem and the final product does not contain agrochemical residues. This option combines and takes advantage of the advantages that the plants offer, through its components and active properties that are effective for the manufacture of insecticides. Because of their high effectiveness in controlling tropical diseases, insects and high yield per hectare, composition and mode of action, industrial insecticides such as oxamyl, cypermethrin, busan-30 are frequently used, however they have an adverse, effect studies show that uncontrolled and unconscious use, causes long-term cancer, leukemia, damage to the nervous system, respiratory
\end{abstract}


Bordones (et al): Comparación de la efectividad en la protección de cultivos de tomates con insecticidas orgánicos a base de: ajo (allium sativum) y Nim (azadirachta indica)

and lung conditions; by inadequate prevention measures of the manipulator, becomes a public health problem in which the state has to invest more in medicines and treatments.

The tomato is one of the items produced in the region, its production costs are low, it has high nutritional quality in vitamins and minerals. In its stages of development affected by pests such as miners, suckers, chewers, etc. and is attacked by various diseases because the seedlings are an open field and its harvest period is very long ( 3 to 5 months). The mode of action of both insecticides in these pests is by ingestion and inhalation.

The main substance present in the extract of the neem leaves is Azadirachtin which has a repellent action against insects pest and also breaks the life cycle preventing its multiplication [1], in the case of garlic, its main active substance for processing of insecticides is the Alina that when released interacts with an enzyme called alinase and generates allicin which is the substance that generates the characteristic smell of garlic and with which the insects are repelled from the infected plant. [2]

The processing of these insecticides began with the processing and extraction of substances from two products; later applied in the seedlings.

After the application of the insecticides it was observed that the plants affected by the insects, improved their shape and had a better growth. The insecticide based on Nim had a 100\% effectiveness in the seedlings where it was tested. On the other hand, the effectiveness of garlic was $66.7 \%$ in the seedlings treated, the neem mode of action in the plant is introduced to the sap and of the garlic through its roots, we conclude that it is the best product for the production of organic insecticides.

Keywords Nim, insecticide, ecological agriculture, toxic, azadirachtin, pests, insects, allicin, alinase.

* Corresponding author: alma.chen@utp.ac.pa

\section{Introducción}

Los insectos plagas pueden reducir significativamente el rendimiento de los cultivos y su calidad, cuando se observa el ataque se debe actuar de inmediato, la mejor manera de controlar las plagas es con el uso de insecticidas que sean de gran absorción y actuación inminente, independiente de que sean orgánicos e inorgánicos. La aparición de plagas y enfermedades están asociadas al ambiente en el que están, al cuidado que se le da y la variedad del cultivo. El conocimiento de la plaga, permitirá tener un mejor control y prevención en el cultivo.

Los insecticidas orgánicos son una mejor opción para el control de las plagas en los cultivos ya que no son perjudiciales para la salud tanto del consumidor como del productor y de igual manera no tienen un efecto dañino contra el medio ambiente.

Tanto los insectos plagas como los hongos provocan un deterioro en los plantones de tomates y por consiguiente le causan la muerte. Algunos de estos insectos desarrollan su metamorfosis en la planta, a medida que van evolucionando se van alimentando de ella, causando debilitamiento, marchitamiento, crecimiento detenido y lento.

El ajo es un repelente sistémico de alto espectro y rico en azufre; el Nim tiene efectos repelentes, insecticidas y antihormonales; además tiene cualidades terapéuticas. La importancia de ambos es que funcionan por ingestión convirtiéndose así en excelentes insecticidas.

Los insecticidas orgánicos se fabrican evitando usar productos químicos nocivos. Una ventaja de los insecticidas orgánicos es también que se puede hacer pesticidas orgánicos desde su propia casa. Esta es rentable y saludable también para el medio ambiente [4].

Esta investigación consiste en la elaboración de dos insecticidas orgánicos a bases de ajo y de Nim, posteriormente se realizará una comparación para determinar la efectividad en el control y eliminación de insectos en las plantaciones de tomate. Los plantones estuvieron a campo abierto, en parcelas separadas que permitieran observar y aplicar mejor el insecticida. Los intervalos de aplicación de ambos dependen de su duración y se realizará durante un mes.

Para la elaboración, los procedimientos se hicieron por separado y su aplicación igual, para así seleccionar el insecticida más efectivo y menos perjudicial para la salud.

\section{Materiales y métodos}

La materia prima utilizada fue: ajo, hojas de Nim y agua utilizada como disolvente. Los materiales que se usaron para la elaboración de los productos fueron: una olla de cocina, licuadora, mortero, pistilo y atomizadores para poner a prueba nuestros productos. El procedimiento usado para el desarrollo de nuestra investigación lo vemos a continuación.

\subsection{Obtención y procesamiento de la materia prima}

Para la elaboración de insecticida a base de ajo se procedió a obtener las cabezas de ajo, posteriormente se maceró el ajo, para luego mezclarlo con un litro de agua. Posteriormente, se dejó en reposo aproximadamente 24 horas, después se calentó en una hoya de cocina durante 5 minutos, 
Bordones (et al): Comparación de la efectividad en la protección de cultivos de tomates con insecticidas orgánicos a base de: ajo (allium sativum) y Nim (azadirachta indica)

finalmente se colocó en reposo durante 24 horas más. La mezcla se tamizó y la solución del extracto de ajo se colocó en un atomizador. Finalmente se aplicó en las plantas de tomate como se muestra en la figura1.

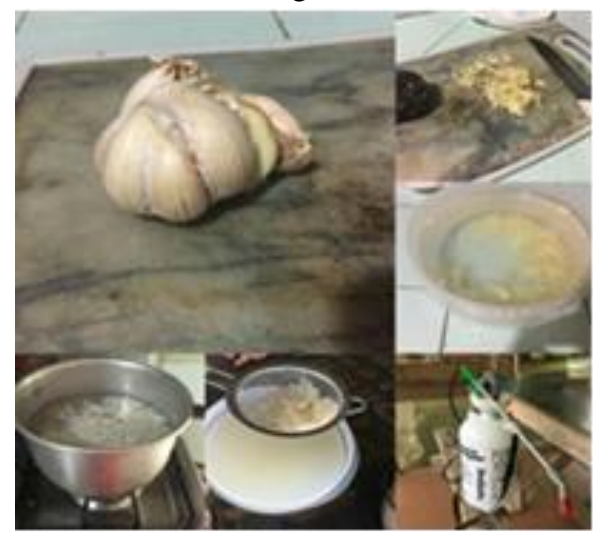

Figura 1. Procesos de preparación del insecticida de ajo.

Para la elaboración del insecticida a base de Nim se recolectaron las hojas del árbol de Nim, estas se dejaron en agua por un tiempo aproximado de 48 horas. Posteriormente se colocaron en una licuadora en donde fueron trituradas, el producto fue tamizado y vertido en un envase, el cual se aforó con el agua y se dejó en reposo por 48 horas. El líquido obtenido se colocó en un atomizador y se procedió a fumigar las plantas afectadas.

Tabla 1. Cantidades utilizadas

\begin{tabular}{|c|c|c|c|}
\hline \multirow{2}{*}{$\begin{array}{c}\text { Materia } \\
\text { prima }\end{array}$} & $\begin{array}{c}|c| \\
\text { Cantidad } \\
(\mathbf{g})\end{array}$ & $\begin{array}{c}\text { Cantidad de } \\
\text { disolvente } \\
\left(\mathbf{H}_{2} \mathbf{O}\right)(\mathbf{L})\end{array}$ & $\begin{array}{c}\text { Producto } \\
\text { obtenido } \\
\text { (litros) }\end{array}$ \\
\hline Nim & 38.00 & 1 & 1.038 \\
\hline Ajo & 33.00 & 1 & 1.033 \\
\hline
\end{tabular}

\section{Resultados y discusión}

Ambos insecticidas funcionan con efectividad; y pueden ser utilizados. Sus beneficios en el cultivo son excelentes, según los datos obtenidos en nuestro estudio.

En la práctica de campo se demostró que los plantones de las diferentes parcelas y en los diferentes días en los cuales se aplicaron los insecticidas, dieron como resultado, que el insecticida elaborado a base de Nim es más eficiente, debido a que su protección en la planta es más duradero. Esta no presentaba, daños de insectos y evitaban acercarse a la planta debido al olor del repelente. Esto los observamos en la tabla 2 y la figura 2.

\subsection{Efectividad del Nim}

Tabla 2. Estados del plantón con el insecticida de Nim

\begin{tabular}{|c|c|c|c|c|}
\hline $\begin{array}{c}\text { Plantones } \\
\text { de } \\
\text { tomate }\end{array}$ & Color & $\begin{array}{c}\text { Cantidad } \\
\text { de hojas }\end{array}$ & Plagas & $\begin{array}{c}\text { Duración } \\
\text { del } \\
\text { insecticida }\end{array}$ \\
\hline Antes & $\begin{array}{c}\text { Verde } \\
\text { claro }\end{array}$ & 5 & $\begin{array}{c}\text { Moscas } \\
\text { y } \\
\text { hormigas }\end{array}$ & $\begin{array}{c}15 \text { días } \\
\text { aprox. }\end{array}$ \\
\hline Después & Verde & 6 & Limpio & \multicolumn{2}{|c|}{} \\
\cline { 1 - 3 } & & &
\end{tabular}

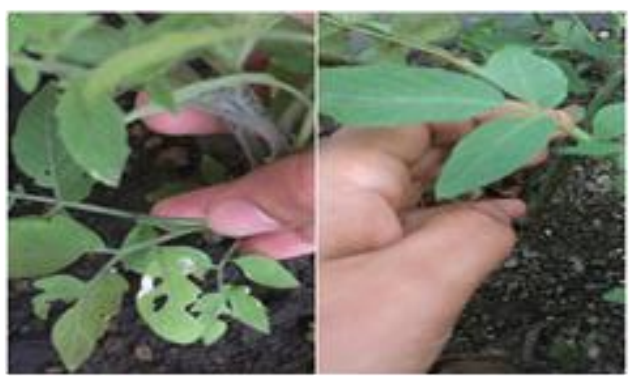

Figura 2. Sin aplicación/Con aplicación.

La tabla 2 nos muestra que antes de la aplicación del insecticida existían insectos plagas, posteriormente a la aplicación, en un tiempo de 15 días no había presencia de insectos plagas y esto lo observamos con el aumento en el número de hojas. Ver figura 2.

\subsection{Efectividad del ajo}

Tabla 3. Estados del plantón con el insecticida de ajo

\begin{tabular}{|l|l|l|}
\hline $\begin{array}{l}\text { Plantones } \\
\text { de tomate }\end{array}$ & \multicolumn{1}{|c|}{ Antes } & \multicolumn{1}{c|}{ Después } \\
\hline Color & Amarillento & Verdoso \\
\hline $\begin{array}{l}\text { Cantidad } \\
\text { de hojas }\end{array}$ & 6 & 5 \\
\hline Plagas & $\begin{array}{l}\text { Hormigas y } \\
\text { gusanos }\end{array}$ & $\begin{array}{l}\text { Hormigas en } \\
\text { pequeñas cantidades }\end{array}$ \\
\hline $\begin{array}{l}\text { Duración } \\
\text { del } \\
\text { insecticida }\end{array}$ & $\begin{array}{l}\text { 8 días } \\
\text { aproximadamente }\end{array}$ & \\
\hline
\end{tabular}

En cuanto al efecto del ajo, la tabla 3 nos muestra que la efectividad fue por un tiempo de 8 días, donde observamos la disminución de insectos plaga. Finalmente la planta fue atacada y se observa en el número de hojas sanas. Ver figura3. 
Bordones (et al): Comparación de la efectividad en la protección de cultivos de tomates con insecticidas orgánicos a base de: ajo (allium sativum) y $\mathrm{Nim}$ (azadirachta indica)

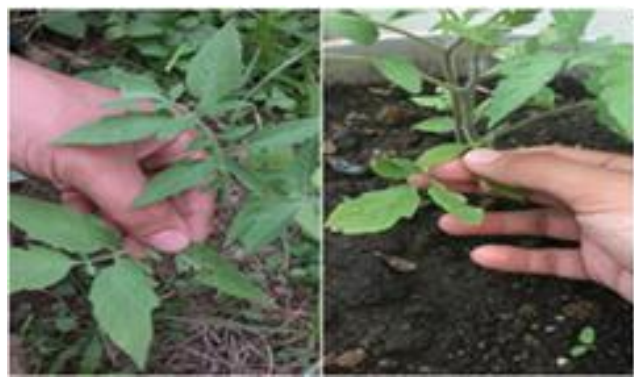

Figura 3. Sin aplicación/con aplicación.

\subsection{Comparación de la efectividad de los insecticidas desarrollados}

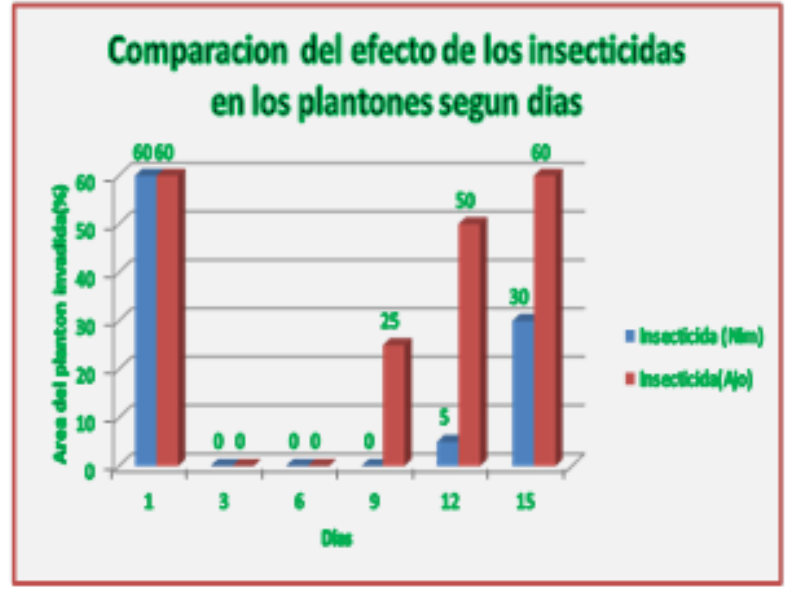

Grafica 1. Plantones afectados vs días.

En cuanto a la comparación de la efectividad de la acción de los insecticidas en las plantas a través del tiempo.

La gráfica 1 nos muestra la comparación de dos insecticidas en el control de insectos en los plantones de tomate, a través de la observación en intervalos de tres días. Luego de aplicado el producto, la presencia de insectos en los plantones disminuyo grandemente por lo cual el plantón presento un mejor estado físico; como se presenta en la gráfica. La gráfica presenta un comportamiento decreciente y luego crece en el momento que el efecto del insecticida pierde su pureza, esto permite a los insectos nuevamente invadir el plantón. El efecto del Nim perdura aproximadamente 15 días y el del ajo 8 días. La aplicación del producto se hizo a plantas diferentes, en el mismo.

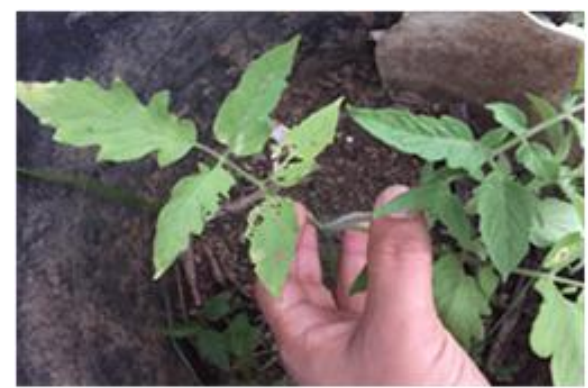

Figura 4. Plantón de tomate afectado por insectos.

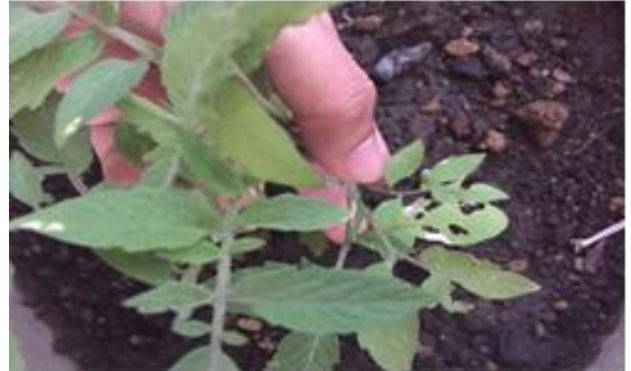

Figura 5. Plantón de tomate afectado por insectos.

\section{Conclusiones}

Los resultados de este estudio aportan información valiosa sobre el uso de los insecticidas orgánicos, queda demostrado que el insecticida a base de hojas de Nim, con alto contenido de Azadiractina, presenta una gran efectividad de eliminación de insectos y protección del cultivo.

\section{SUGERENCIAS}

Recomendamos el uso del insecticida a base de Nim a los productores de cultivos de tomates u otros rubros por su efectividad y bajo costo además por ser un producto amigable con la salud y el medio ambiente.

\section{RECONOCIMIENTOS}

Agradecemos a Dios por darnos salud y sabiduría para desarrollar nuestro proyecto y terminarlo con éxito. A nuestros padres y amigos por brindarnos el apoyo incondicional en todo momento para lograr nuestros objetivos.

\section{REFERENCIAS}

[1] Gimeno, Juan. EL NIM, UN INSECTICIDA NATURAL. EL NIM, UN INSECTICIDA NATURAL. [En línea] 4 de mayo de 2006. [Citado el: 9 de junio de 2017.] http://ecomaria.com/blog/el-Nim-un-insecticida-natural/..

[2] EL USO DEL AJO COMO REPELENTE DE PLAGAS INSECTOS Y COMO CONTROL DE ENFERMEDADES CRIPTOGÁMICAS. EL USO DEL AJO COMO REPELENTE DE PLAGAS INSECTOS Y COMO CONTROL DE ENFERMEDADES CRIPTOGÁMICAS. [En línea] 17 de junio de 2008. [Citado el: 9 de junio de 2017.] http://ecomaria.com/blog/el-uso-del-ajo-como-repelente-deplagas-insectos-y-como-control-de-enfermedadescriptogamicas/.

[3] marketing, arabuko. La importancia de los insecticidas en el campo agrícola. La importancia de los insecticidas en el campo agrícola. [En línea] 2017. [Citado el: 18 de junio de 2017.] http://arabuko.mx/la-importancia-de-los-insecticidasen-el-campo-agricola/.

[4] Christopher. Importancia de los Insecticidas Organicos. Importancia de los Insecticidas Organicos. [En línea] 3 de noviembre de 2010. [Citado el: 18 de junio de 2017.] http://agronomiaitec.blogspot.com/2010/11/importancia-delos-insecticidas.html. 